\title{
Characteristics and magnetic field properties of the Hamamatsu R11265 Multi-Anode Photomultiplier
} Tubes

\author{
Haofei Luo* and Stephan Eisenhardt, Adam Morris, Matthew Needham, Josh Neill \\ The Univerisity of Edinburgh \\ E-mail: haofei.luoded.ac.uk
}

The LHCb detector will be upgraded in 2018 to allow the experiment to run at a luminosity of 2 $\times 10^{33} \mathrm{~cm}^{2} \mathrm{~s}^{-1}$. Since the experiment will be read out at the crossing rate of $40 \mathrm{MHz}$, the Hybrid Photon Detectors of the Ring Imaging Cherenkov detectors will need to be replaced. We report on studies of the Hamamatsu model R11265 Multianode Photomultiplier as part of the effort to qualify their use in the LHCb upgrade. Comparisons with the model R7600 are also made. Due to the LHCb bending magnet, the MaPMTs must operate at a residual fringe field of up to a maximum of 25 Gauss. The behaviour of the MaPMTs in a magnetic field is studied and field shielding is developed.

The 15th International Conference on B-Physics at Frontier Machines at the University of Edinburgh, 14 -18 July, 2014

University of Edinburgh, UK

\footnotetext{
* Speaker.
} 


\section{Introduction}

The LHCb upgrade will take place after the Second Long Shutdown (LS2) of the LHC. At that time LHCb will have accumulated a dataset corresponding to an integrated luminosity of around $8 \mathrm{fb}^{-1}$, delivered at a luminosity of $4 \times 10^{32} \mathrm{~cm}^{-2} \mathrm{~s}^{-1}$ [1]. At that point it will be unprofitable to continue running $\mathrm{LHCb}$ at the same luminosity, due to the slow increase of statistical precision. The upgraded experiment will enable an operating luminosity of around $2 \times 10^{33} \mathrm{~cm}^{-2} \mathrm{~s}^{-1}$ which requires $40 \mathrm{MHz}$ readout of all sub-detectors.

The Hybrid Photon Detectors (HPDs) [2] currently used in the LHCb Ring Imaging Cherenkov Detectors (RICH) [3] have an embedded chip limited to a $1 \mathrm{MHz}$ readout rate [4]. Therefore the HPDs need to be replaced. The baseline for the new photon detector is the Multianode Photomultiplier R11265 from Hamamatsu [1]. The LHCb dipole magnet, used for the momentum measurement of charged particles, results in a residual fringe field at the photon detector plane of RICH-1 of up to 25 Gauss, even with global shielding. For operation in LHCb, the behaviour of the photon detectors in a magnetic field is critical [5].

\section{MaPMT}

The latest generation of MaPMT, the Hamamatsu R11265 has been characterised, as well as the old generation, prototype R7600 MaPMT. Figure 1 shows a view through the entry windows of both models. The MaPMT consists of an array of square anodes $(8 \times 8)$ each with its own metal dynode chain incorporated into a single vacuum tube. The pixel size of R11265 and R7600 are 2.9 $\times 2.9 \mathrm{~mm}^{2}$ and $2 \times 2 \mathrm{~mm}^{2}$ respectively. With a very small inactive border, the active area fraction of R11265 is 77\%, a significant increase from 50\% provided by the R7600. The thickness of the UV glass window is $0.8 \mathrm{~mm}$ and the photocathode material is Bialkali which has a quantum efficiency of $20 \%$ at $400 \mathrm{~nm}$. At an operating voltage of $1 \mathrm{kV}$, the gain of a single photoelectron is expected to be of order $10^{6}$.
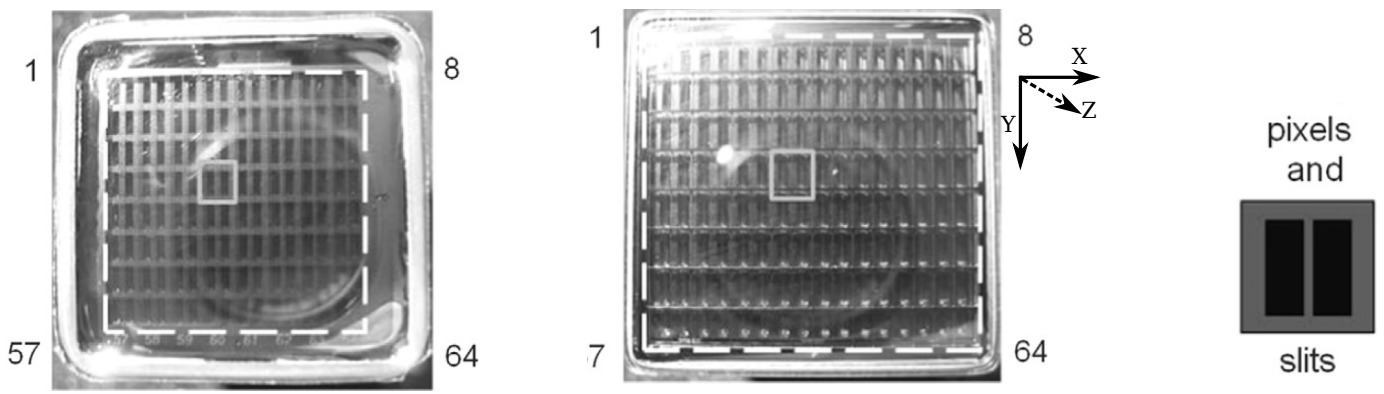

Figure 1: Entry windows of the R7600 (left) and the R11265 (middle), with an indication of active area (dashed line) and one pixel (solid line) shown on the right.

\section{Test setup}

A single-photon MaPMT testing system has been set up in the laboratory in Edinburgh. It consists of a pulsed LED light source, xy-stage stepper motor and data acquisition system. Figure 2 
shows the flow chart of the system. The pulsed LED light with a wavelength of $470 \mathrm{~nm}$ is delivered via a light guiding fibre and focused to a spot size of $\sim 0.1 \mathrm{~mm}$ using a graded index lens to illuminate the MaPMT. The fibre end and lens are mounted on the xy-stage stepper motor and illuminate one pixel at a time, see Figure 3. The entire setup is encapsulated in a dark and electrically shielded box. To match the dynamic range of the subsequent electronics, the signal pulses from the MaPMT are amplified by a factor $\times 100$, using a sequence of two linear amplifiers per channel, each with a bandwidth of $350 \mathrm{MHz}$ and individual gains of $\times 10$. Another channel of the pulse produces a $\sim 35 \mathrm{~ns}$ integral gate which is synchronised with the MaPMT signal. The data acquisition system (CAEN V792) then integrates the signal. Due to the limited number of available amplifiers, only 16 MaPMT channels are recorded at the same time. The unconnected pixels are grounded to prevent internal charge-up of the anodes.
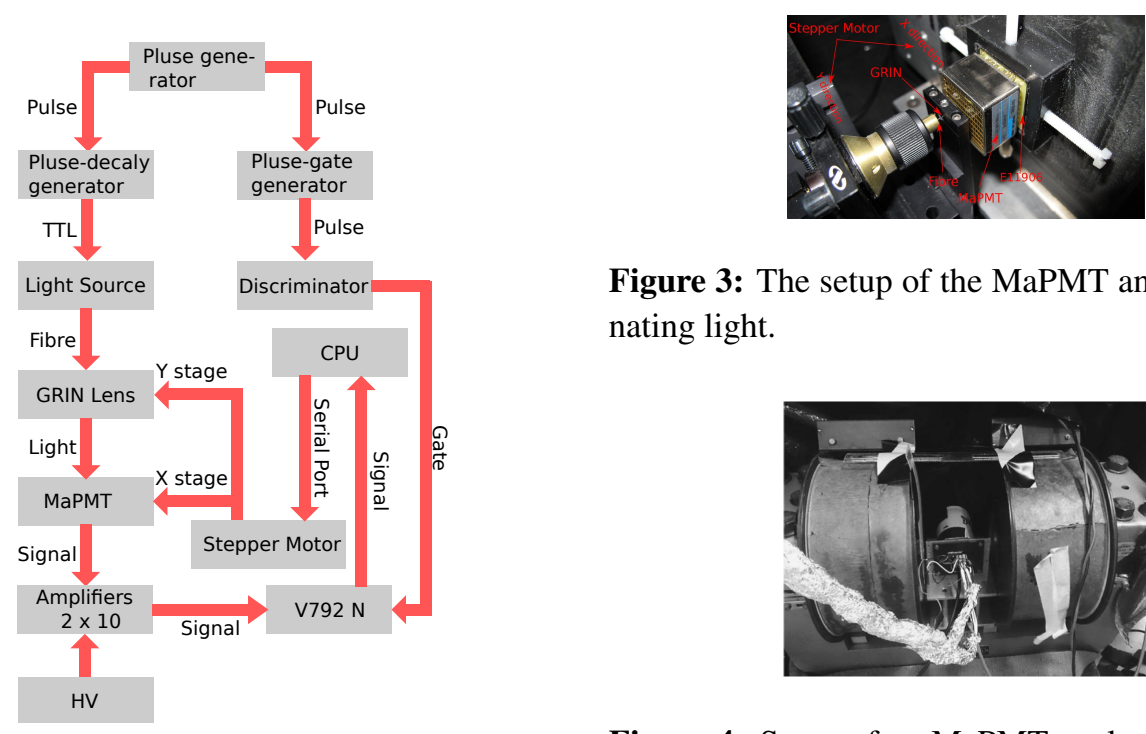

Figure 3: The setup of the MaPMT and the illuminating light.

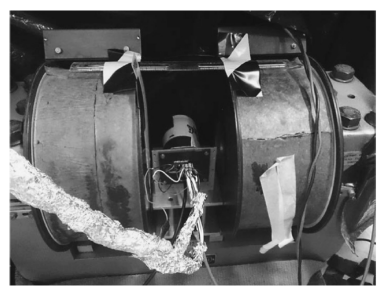

Figure 4: Setup of an MaPMT enclosure mounted

Figure 2: Flow chart of the MaPMT testing system. between the pole shoes of the magnet.

As an alternative setup, we can mount the MaPMT in a small dark cylinder, which fits into the opening of a dipole magnet, delivering fields up to $300 \mathrm{G}$, see Figure 4. In this small cylinder the pulsed light is delivered by a light guide entering from the back and the light reflects diffusely off white paper at the front part of the cylinder. This results in a fairly homogeneous illumination of the whole active area of the MaPMT. The intensity of the light source is regulated to produce spectra which are dominated by single photoelectron signals, as shown in Figure 5.

\section{MaPMT characterisation and response to magnetic fields}

The signal in one channel is amplified by secondary electron generation at the cascaded dynodes. Ideally, the electrons are confined to one channel as the pixels do not share the cascading stages. Due to geometric variation of the dynode and/or small nonuniformities of the electric field, electrons have a small probability to pass the boundary to a neighbouring cascade when travelling to the next dynode [6]. They get further multiplied in the other cascade and generate a cross talk signal in the neighbouring pixel. 

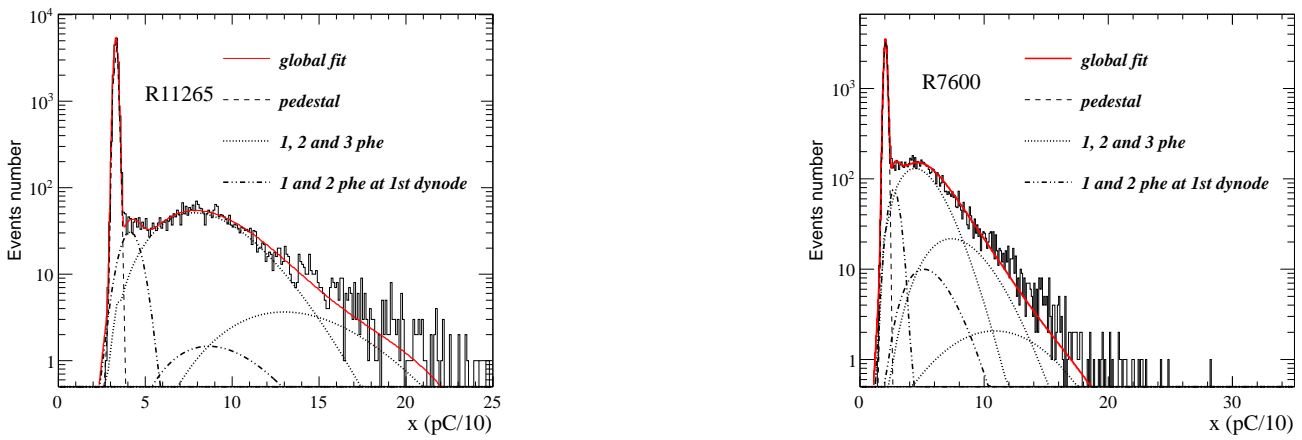

Figure 5: Typical data spectra and fit results of the MaPMT model R11265 and R7600. The high voltages are $950 \mathrm{~V}$ and $900 \mathrm{~V}$ respectively. Legend 1,2 and 3 phe correspond to the signal shapes originating from 1 , 2 and 3 photoelectrons, respectively.
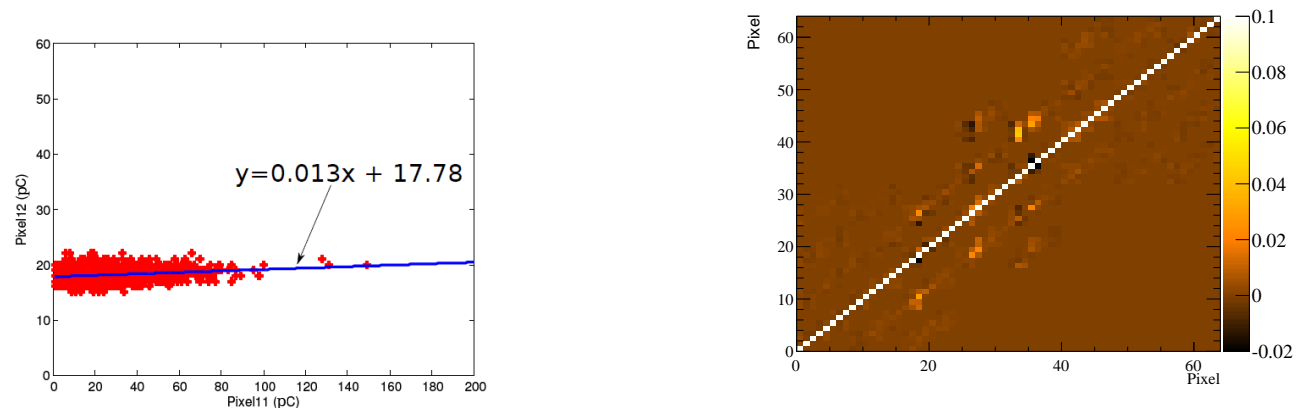

Figure 6: Left: a scatter plot of charge pulses in two neighbouring pixels of R11265 without external magnetic field; Right: the cross talk map of R11265 device. The slope of the left plot is the cross talk of two pixels and figure on the right shows the fit slope with in on MaPMT.

Figure 6 shows the correlation between charge pulses of two adjacent pixels, of which only one is illuminated. Each data point represents the recorded charges for one light pulse without external magnetic field. The amount of charge found in a neighbouring channel due to cross talk is expected to be linearly related to the charge produced in the illuminated pixel. Thus, the fitted slope of the distribution is a measure of the strength of the cross talk. The R11265 shows a cross talk of $<2 \%$ to the direct neighbours and of $<1 \%$ to corner neighbours. Integrated over all eight neighbours the typical cross talk is $\sim 8 \%$.

With increasing high voltage, more secondary electrons are produced when the accelerated electrons hit the next dynode. Figure 7 shows the signal size exponentially increases with high voltage and doubles every $60 \mathrm{~V}$. Conversely the signal loss, calculated by fitting the signal spectra and integrating over the signal contributions to a cut $5 \sigma_{\text {noise }}$ above the pedestal, exponentially decreases with high voltage.

Figure 8 shows the relative efficiency to detect single photoelectrons,normalized to unity at $\mathrm{B}=0 \mathrm{G}$, as a function of magnetic field strength. The efficiency is determined from the count of photons above a $5 \sigma_{\text {noise }}$ threshold. Here the data are shown for the R7600 and the R11265 with $\mathrm{HV}=900 \mathrm{~V}$ and for the R11265 with HV=1000V. The Both MaPMTs show the strongest sensitivity 

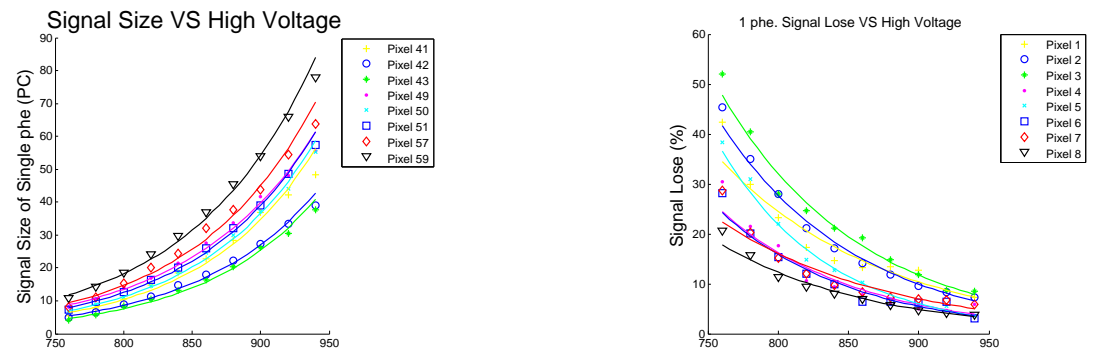

Figure 7: Single photoelectron signal size (left) and signal loss (right) of 8 pixels as a function of high voltage.

to the magnetic field in the longitudinal direction (z) and also show a higher sensitivity in the $\mathrm{y}$ direction, defined in Figure 1. But the R11265 in general is more sensitive to magnetic fields than the R7600. This is due to the geometric changes to optimise the active area. It is also seen that at low gain the loss in efficiency due to magnetic fields is greater. Figure 9 displays the magnetic field strengths at which the single photoelectron efficiency drops below $90 \%$ for each pixel. The larger the value, the less sensitive the pixel is to magnetic field. It becomes clear that the edge columns along the direction of the entry slits are most sensitive within the device.
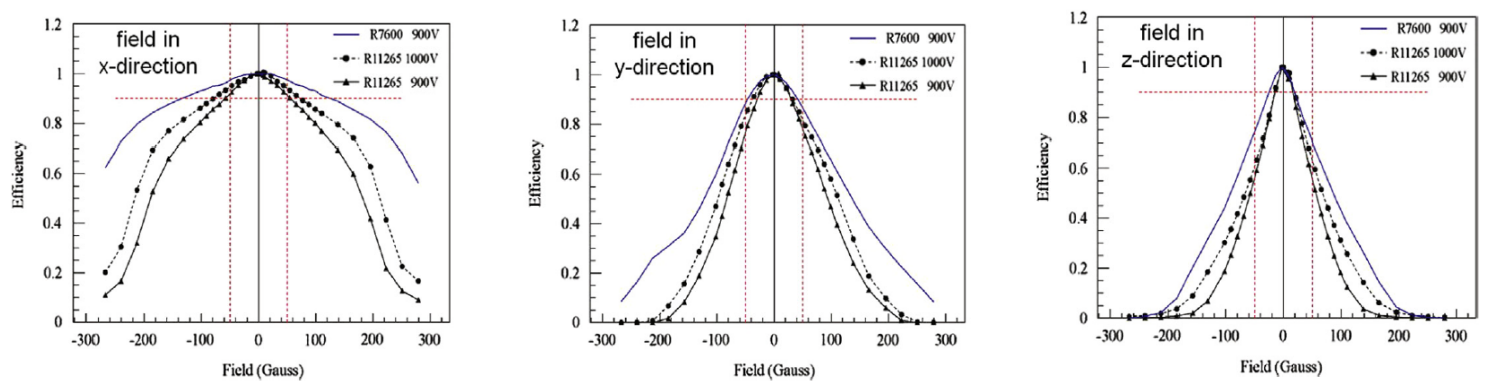

Figure 8: Single photoelectron relative efficiency of the R7600 and R11265 MaPMT as a function of magnetic fields along the three axes.
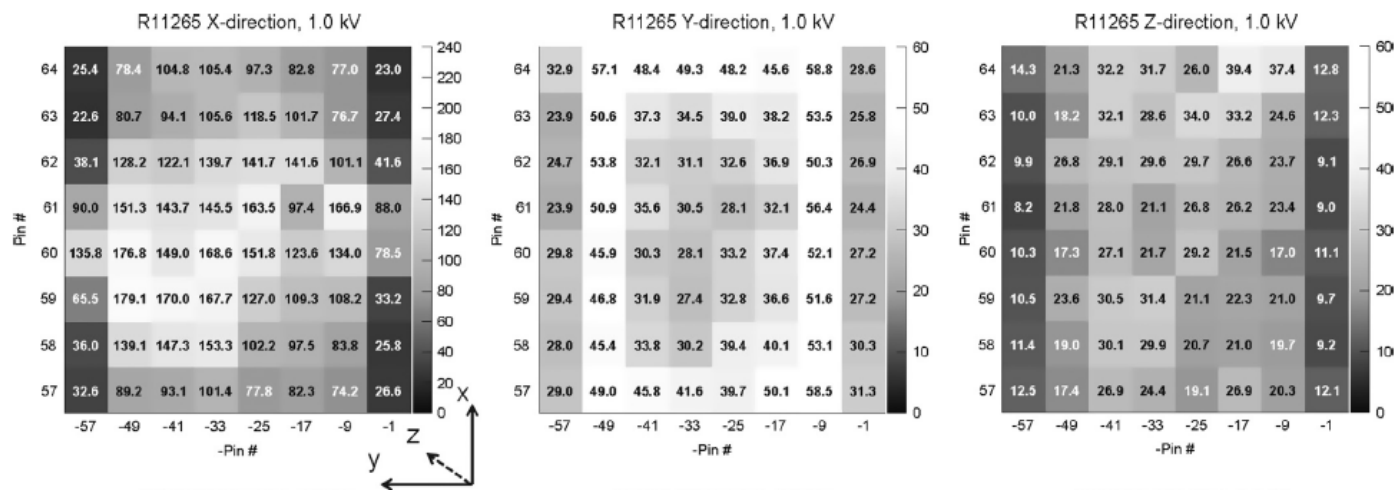

Figure 9: Magnetic field strength maps for 90\% single photoelectron efficiency of the R11265 MaPMT. 


\section{Mu-metal shielding}

To reduce the effect of the residual fringe field of the LHCb dipole magnet Mu-metal shields are needed. Several prototype shields have been made and tested. Figure 10 shows the protrusion length of the Mu-metal from the MaPMT UV glass surface and the corresponding single photoelectron efficiency above a $5 \sigma_{\text {noise }}$ threshold. Longer protrusion leads to the higher photoelectron efficiency at the expense of shadowing of light incident off-axis.For the protrusion length of $12 \mathrm{~mm}$, the Mu-metal can effectively shield the residual magnet field.
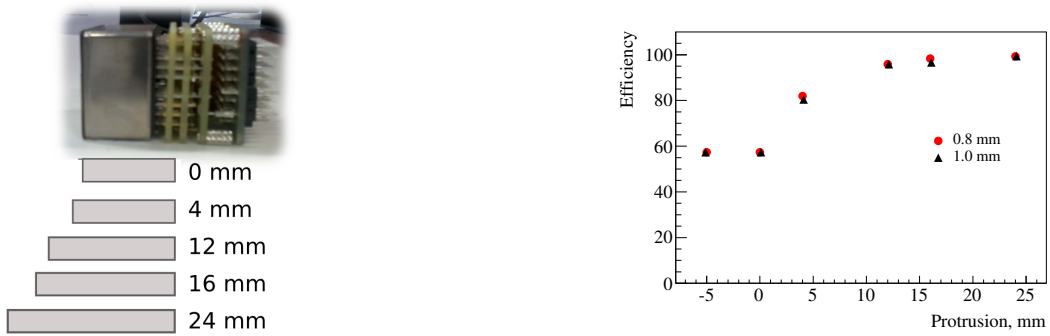

Figure 10: The protrusion length of Mu-metal from the UV glass surface (left), and the single photoelectron efficiency corresponding to different protrusion lengths (right). The $0.8 \mathrm{~mm}$ and $1 \mathrm{~mm}$ points represent the thickness of the Mu-metal and the magnet field B is $30 \mathrm{mT}$.

\section{Conclusion}

The characteristics of the R11265 MaPMT have been measured. Single photons can be clearly separated from the noise and the measured cross talk is at an acceptable level for the LHCb upgrade. The signal size of the R11265 increases exponentially with high voltage and doubles every 60V. The R11265 MaPMT is more sensitive to magnet field than the R7600 MaPMT. The largest effects on the signal efficiency are seen for magnet fields along the z-direction, and the MaPMTs are most robust against fields oriented along the $\mathrm{x}$-direction. Mu-metal can be used to effectively shield the MaPMTs from residual fringe field of the LHCb dipole magnet.

\section{References}

[1] LHCb Collaboration, LHCb PID Upgrade Technical Design Report, [CERN-LHCC-2013-022. LHCB-TDR-014]

[2] M. Alemi, First operation of a hybrid photon detector prototype with electrostatic cross-focussing and integrated silicon pixel readout, Nuclear Instruments and Methods in Physics Research Section A, 449 (2000) 48

[3] LHCb Collaboration, The LHCb Detector at the LHC, Journal of Instrumentation, 3 (2008) S08005

[4] K.Wyllie, A pixel readout chip for tracking at ALICE and particle identification at LHCb, in proceedings of the Fifth Workshop on LHC Electronics

[5] S.Eisenhardt, Characterisation and magnetic field properties of multianode photomultiplier tubes, Nuclear Instruments and Methods in Physics Research Section A

[6] C.Arnaboldi, Cross talk study to the single photon response of a flat panel PMT for the RICH upgrade at LHCb, IEEE Nucl.Sci.Symp.Conf.Rec., 1513, (2009) 